\title{
Photoluminescence Emission Control of Porous Silicon
}

\author{
Marwa Nabil \\ Advanced Technology and New Materials Research Institute, City of Scientific Research and Technology Applications, \\ New Borg El-Arab City, Alexandria, Egypt \\ Email: marwa.nabil3010@gmail.com
}

How to cite this paper: Nabil, M. (2019) Photoluminescence Emission Control of Porous Silicon. Soft Nanoscience Letters, 9, $35-44$.

https://doi.org/10.4236/snl.2019.93003

Received: June 17, 2019

Accepted: July 27, 2019

Published: July 30, 2019

Copyright (c) 2019 by author(s) and Scientific Research Publishing Inc. This work is licensed under the Creative Commons Attribution International License (CC BY 4.0).

http://creativecommons.org/licenses/by/4.0/

\section{(c) (i) Open Access}

\begin{abstract}
This paper reports the feasibility of synthesis and characterization of nano-porous silicon (NPS) powder and (Nickel/nano-porous silicon, Ni/NPS) nano-composite prepared using dual techniques (alkaline chemical etching process and ultra-sonication technique). The structural and the optical properties of the fabricated structures are inspected using X-ray Diffraction, Fourier Transform Infrared Spectrophotometer, Raman Spectroscopy, and Fluorescence Spectrophotometer Photoluminescence. All the results have agreed that NPS is one of the most suitable materials used as active material in the LED fabrication; by changing the main factors in the preparation process, so the different physical and chemical properties are obtained. NPS produces two emission regions that correspond to orange-red and dark red; on the other hand, (Ni/NPS) produce the yellow emission. So, the photoluminescence emission is controllable by adjusting the preparation conditions. The optical data recorded here are useful for the production of the nanoscale optical devices.
\end{abstract}

\section{Keywords}

Optical Materials, Chemical Synthesis, Optical Properties

\section{Introduction}

During recent years, many materials have come to the attention of researchers, using as light emitting device (LED), due to the extensive commercial demand. The most popular composites are InGaN, GaAs and AlGaN, that ascribed to the satisfactory emission intensity and mature fabrication techniques. However, it is a challenge against these materials for the incompatibility with Si-based microelectronics process and the expensive cost for commercial demand. Naturally, 
LEDs made from Si which is easily inserted into chips resulting translation with high speed. So far no other materials achieve perfect commercial applications as $\mathrm{Si}$. But, $\mathrm{Si}$ is hardly photoluminescence emission with respect to other materials, such as InGaAs, as a result of its indirect band gap [1]. On the other hand, the finding of photoluminescence in porous silicon has sparked the hope for silicon as a material also for optoelectronic applications. Until today, photoluminescence from nanostructured silicon could be demonstrated not only from porous silicon, but also silicon nanocrystals formed in $\mathrm{SiO}_{2}$ matrix by implantation of $\mathrm{Si}$ ions and subsequent annealing and isolated silicon nanoparticles [2].

After the monitoring of highly efficient visible photoluminescence (PL) at room temperature from porous silicon (PS) by Canham, this material has become an attractive material in electronics and optoelectronics fields [3]. The PS morphology consists of a silicon nancrystallites (nc-Si) network, which depends on several parameters (i.e. air and storage conditions). The freshly prepared PS is covered by hydrogen and at exposure to ambient air that causes the progressive modification of its surface by oxidation process. It produces modification of nc-Si optical properties. For solvation the structural problem and improving the PL of PS, it is necessary to change the composition of the PS surface by incorporating some elements. The transition metals (i.e. iron, cobalt or nickel) are the solution; it can be used for delegating inside the silicon pores that makes a change of the PS optical properties [4].

Many substitution materials have been suggested for improving Si-luminescence. And, the most widely studied material is PS, which displayed strong photoluminescence (PL) in the visible and infrared region of the optical spectrum [5]. The PS Light emission is a great success for the development of silicon-integrated optoelectronic devices, which is compatible with microelectronic technologies [6].

For this study, we present experimental results that depending on a simple wet alkali chemical etching technique process parallel with using Ultra-sonication mechanism for porous silicon production, strong visible light emission can be achieved. In addition, using a transition element (i.e. Ni), which is changing the PL emission range, as shown in the following.

\section{Materials and Methods}

The dual process of the nano-porous-silicon (NPS) powder preparation was consisted of; the ultra-sonication technique (at room temperature) as a simple way and the alkali chemical etching technique (ACE). The used reagents in the ACE process were; n-propanol, and Potassium hydroxide (KOH). The suitable mass for starting was a $5 \mathrm{~g}$ silicon powder (99\%, Sigma-Aldrich). It was put in an etching solution that contained different $\mathrm{KOH}$ concentrations $(3,4.5$, and 6 weight $\%)$, n-propanol (30 Vol\%), for different sonication times $(2,3,4$, and $5 \mathrm{~h}$ ). The powder produced was filtered, washed, and then dried at $40^{\circ} \mathrm{C}$ overnight. The nano-Ni layers were deposited on the NPS powder by a chemical deposition technique. The prepared NPS powder was immersed in an aqueous $\mathrm{NiCl}_{2}$ solu- 
tion at $80^{\circ} \mathrm{C}$ for 30 minutes. And then, the nanocomposite (Ni/NPS) powder product was filtered out again from the Ni salt solution and dried at $60^{\circ} \mathrm{C}$ overnight for two days.

The NPS powder structure is characterized using XRD (X-ray, using $\mathrm{Cu}-\mathrm{Ka}$ radiation $=1.5405 \AA$ at a scanning rate of $4 \mathrm{~min}^{-1}, 7000$ Schimadzu diffractometer). The formation of the NPS and (Ni/NPS) chemical bonds were determined using FTIR (Fourier Transform Infrared Spectrophotometer, Shimadzu FTIR -8400 s, Japan), Raman Spectroscopy (Senteral-Bruker Raman micro-spectroscopy), and PL (Cary Eclipse Fluorescence Spectrophotometer Photoluminescence, American) analysis of PS, before and after treatment with (Ni).

\section{Results and Discussion}

\section{- X-Ray Diffraction Analysis}

$\mathrm{XRD}$ is selected for its suitability to study the changes of the crystal structure induced by combination between the etching processes and the ultra-sonication technique [7]. Figure 1 shows the XRD patterns of NPS powder. The peaks at $2 \theta^{\circ}$ values $28.23^{\circ}, 47.193^{\circ}, 56.023^{\circ}, 68.989^{\circ}, 76.261^{\circ}, 87.9382^{\circ}$ and $94.8370^{\circ}$ can be indexed as the planes of Si crystals (111), (220), (311), (400), (331), (422), and (511), (JCPDS Card No. 01-079-0613 and 00-027-1402), respectively. All the observed peaks show pure phases of crystalline silicon in face center cubic (FCC) structure. In addition, Figure 1 (c) displays a weak broad diffraction peak at $2 \theta$ $=20^{\circ}$ that corresponds to the thin oxide layer (silica layer), which surrounds the NPS particles [8].

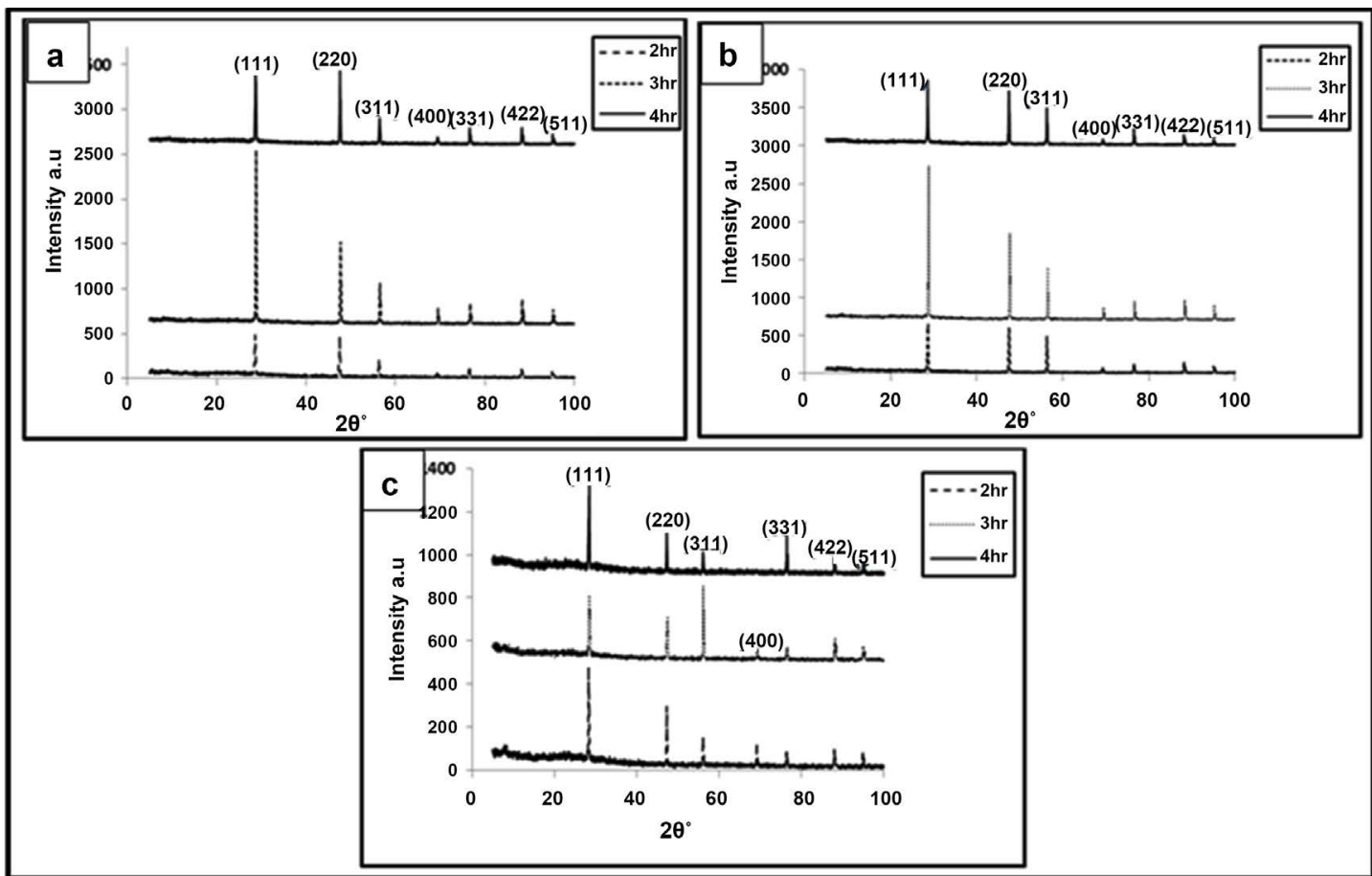

Figure 1. XRD patterns of NPS particles 5 gm of commercial silicon powder; (a) 3 weight\% $\mathrm{KOH}$, (b) 4.5 weight $\% \mathrm{KOH}$, and (c) 6 weight $\% \mathrm{KOH}$. 
The Scherrer's equation is used for calculation of nc-Si average size [9]. Table 1 shows the diffraction peaks of the plane Si (111) at JCPDS card 27-1402. That's due to the (ACE) and the time of the ultra-sonication technique, which produces the intensity changes in three categories of the etchant solution concentrations.

$$
d=\frac{k \lambda}{B \cos \theta}
$$

where $B$ is the FWHM, $K$ is the Scherrer constant $(1>K>0.89), \lambda$ is the wavelength in nanometers, and $d$ the mean crystallite size.

The NPS material energy gap is definitely having higher energy gaps compared with bulk commercial silicon $(1.11 \mathrm{eV})$ [10]. Table 1 shows 2 groups of samples $(4,5,6)$ and $(7,8,9)$, that display the inverse proportional relation between the energy gap value and the NPS crystallize size at the preparation conditions \{the initiated mass of commercial silicon powder $5 \mathrm{gm}$, sonication time (2, 3 , and $4 \mathrm{hr}$ ), at different values of the $\mathrm{KOH}$. It is noticeable sample 9 that the highest value of the energy gap is $2.08 \mathrm{eV}$, which is corresponding to the lowest value of the crystallite size $27.2 \mathrm{~nm}$. So, it refers to the NPS formation with the highest value of porosity. That is a result of the highest value of the energy gap, which corresponds to the increasing of the porosity percent.

As can be seen in Figure 2, the (Ni/NPS) samples showed the diffraction peaks of (PS) at $2 \theta=28.20^{\circ}, 47.20^{\circ}$ and $56.21^{\circ}$ which are corresponding to $\mathrm{Si}$ planes (111), (220) and (211), respectively. In addition, some diffraction peaks at $2 \theta=75.62^{\circ}, 88.26^{\circ}$ and $94.16^{\circ}$, corresponding to the Ni planes (220), (201) and (311), respectively \{JCPDS card 27-1402 and 45-1027, corresponding to Si and $\mathrm{Ni}$, respectively\}. It shows the appearance of Ni planes, that in case of the double percentage of the Ni salt concentration with respect to the presence of a NPS weight percentage (2 Ni: 1 NPS). So, the decreasing of the Si peak intensities is appeared, as a result of the presence of $\mathrm{Ni}$ ions.

\section{- Fourier Transform Infrared Spectrophotometer Analysis}

Table 1. Typical XRD diffraction peaks, crystallite size, and energy gap of NPS powders obtained using different sonication time and etchant concentration ( $\mathrm{KOH}$ weight\%).

\begin{tabular}{ccccc}
\hline Sample number & $\begin{array}{c}\text { KOH concn. } \\
\text { (weight\%) }\end{array}$ & $\begin{array}{c}\text { Sonic. time } \\
(\mathrm{hr})\end{array}$ & $\begin{array}{c}\text { Energy gap } \\
(\mathrm{ev})\end{array}$ & Crystallite size (nm) \\
\hline $\mathbf{1}$ & & 2 & 1.87 & 32.2 \\
2 & 3 & 3 & 1.56 & 47.8 \\
3 & & 4 & 1.75 & 30.1 \\
4 & 4.5 & 2 & 1.83 & 31.5 \\
5 & & 3 & 1.48 & 55.44 \\
6 & & 4 & 1.37 & 63 \\
7 & & 2 & 1.62 & 44 \\
8 & 6 & 3 & 1.92 & 33 \\
9 & & 4 & 2.08 & 27.2 \\
\hline
\end{tabular}




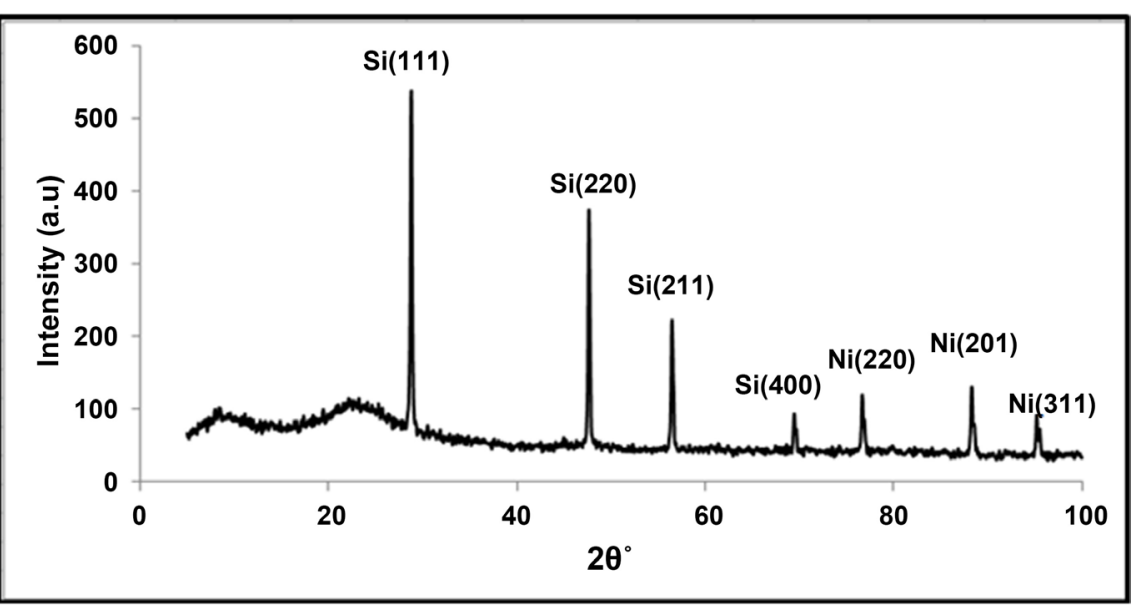

Figure 2. XRD patterns of (Ni/NPS) nano-composite powder.

The presence of various chemical functional groups and formation of NPS are described by the FTIR spectra in the range $400-4000 \mathrm{~cm}^{-1}$. In order to prove the role of wetting agents in the formation of (NPS) FTIR spectra are studied [11]. The FTIR spectrum of the silicon powder after dual reaction; the alkali chemical etching process and the Ultra-sonication technique, is depicted in Figure 3 and Figure 4. The FTIR spectrum band appears in a region $\left(1000-1300 \mathrm{~cm}^{-1}\right)$, which is corresponding to $\mathrm{Si}-\mathrm{O}$ asymmetric stretching in $\mathrm{Si}-\mathrm{O}-\mathrm{Si}$ at 3 weight\% $\mathrm{KOH}$. The characteristic bands almost at 802 and $466 \mathrm{~cm}^{-1}$ are corresponding to the stretching and bending vibrations, respectively. Simultaneously, the spectrum at $1093 \mathrm{~cm}^{-1}$ is appearing in all spectrums at different intensities, which indicates the formation of NPS powder [12]. In addition, the broadband in range (3050 - $3750 \mathrm{~cm}^{-1}$ ) is corresponding to $\mathrm{O}-\mathrm{H}$ stretching modes in $\mathrm{SiOH}$ groups and $\mathrm{H}_{2} \mathrm{O}$. In addition, the spectrum at $1637 \mathrm{~cm}^{-1}$ is due to $\mathrm{O}-\mathrm{H}$ scissor bending vibration in the water. Finally, a weak transmitted band at $\left(2200\right.$ to $\left.2500 \mathrm{~cm}^{-1}\right)$ is observed, which can be attributed to the $\mathrm{O}-\mathrm{Si}-\mathrm{H}$ groups [13].

FTIR measures the change in the NPS chemical composition after an immersion process in $\mathrm{Ni}$-salt solution and the form of $\mathrm{Ni}$ which is deposited on the NPS surface, as shown in Figure 4. The new peak centered at $470 \mathrm{~cm}^{-1}$ which is appeared, generally attributed to $\mathrm{Ni}-\mathrm{O}$ bonds [14]. So, the formation of $\mathrm{NiO}$ is an indication that Ni is deposited on NPS in its metallic form and not in the form of its oxide (Si-O-Ni). Results of FTIR spectra agree with the above XRD data in Figure 2.

\section{- Raman Spectroscopy}

Figure 5 shows the Raman spectra of NPS powder samples started by $5 \mathrm{gm}$ commercial silicon, 6 weight\% $\mathrm{KOH}$ at different sonication times. The Raman spectra have a nearly symmetric peak with different intensities as a result of increasing the sonication time, which produces a direct proportionality relation [15]. The highest spectra intensity appears at wavenumber $518.5 \mathrm{~cm}^{-1}$ that corresponds to sonication time $4 \mathrm{hr}$. It led to peak-shift asymmetry and an enhancement of the peak intensity. 

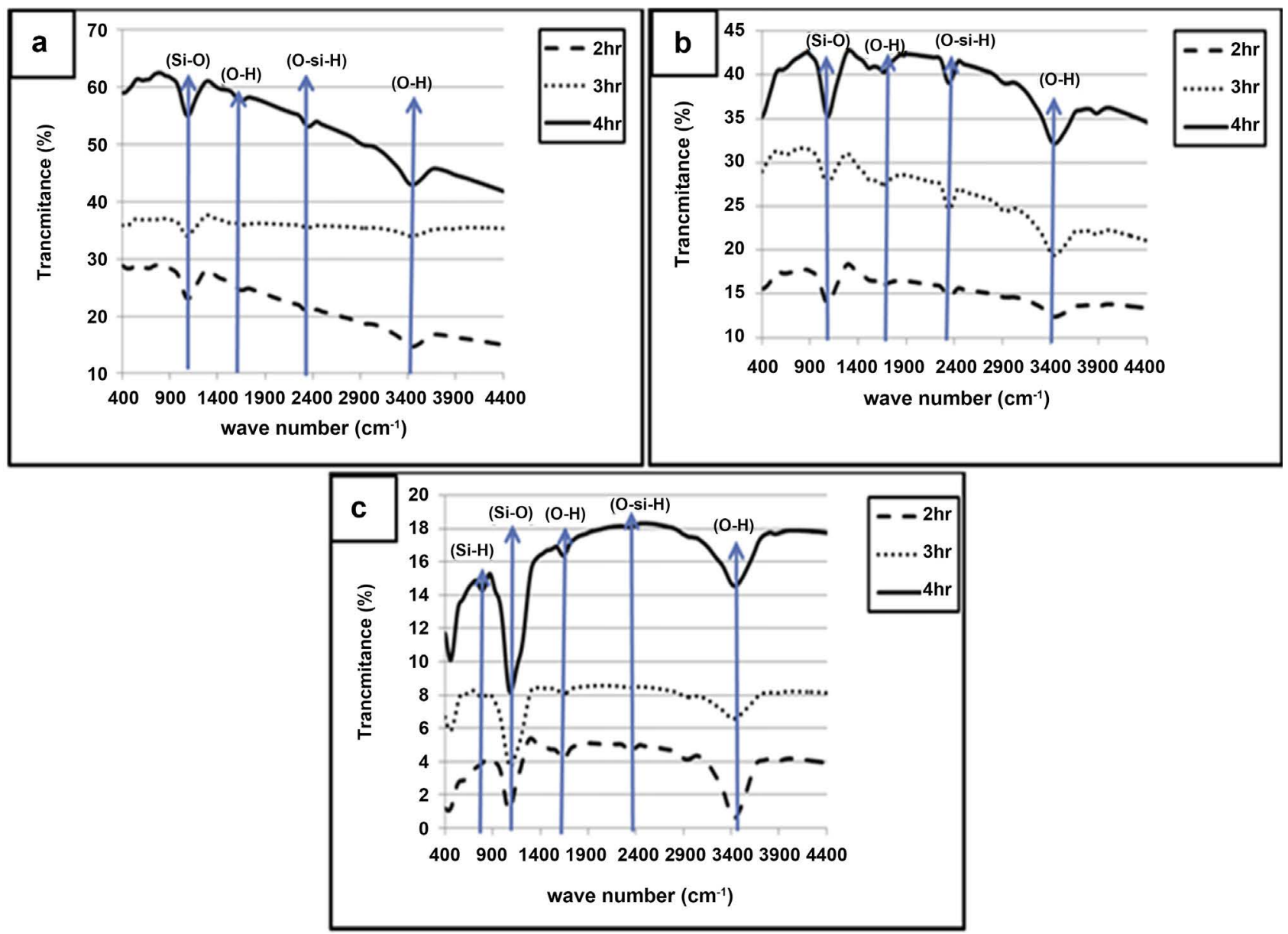

Figure 3. FTIR spectra of NPS powder at the preparation conditions; 5 gm of commercial silicon powder; (a) 3 weight $\% \mathrm{KOH}$, (b) 4.5 weight $\% \mathrm{KOH}$, and (c) 6 weight $\% \mathrm{KOH}$.

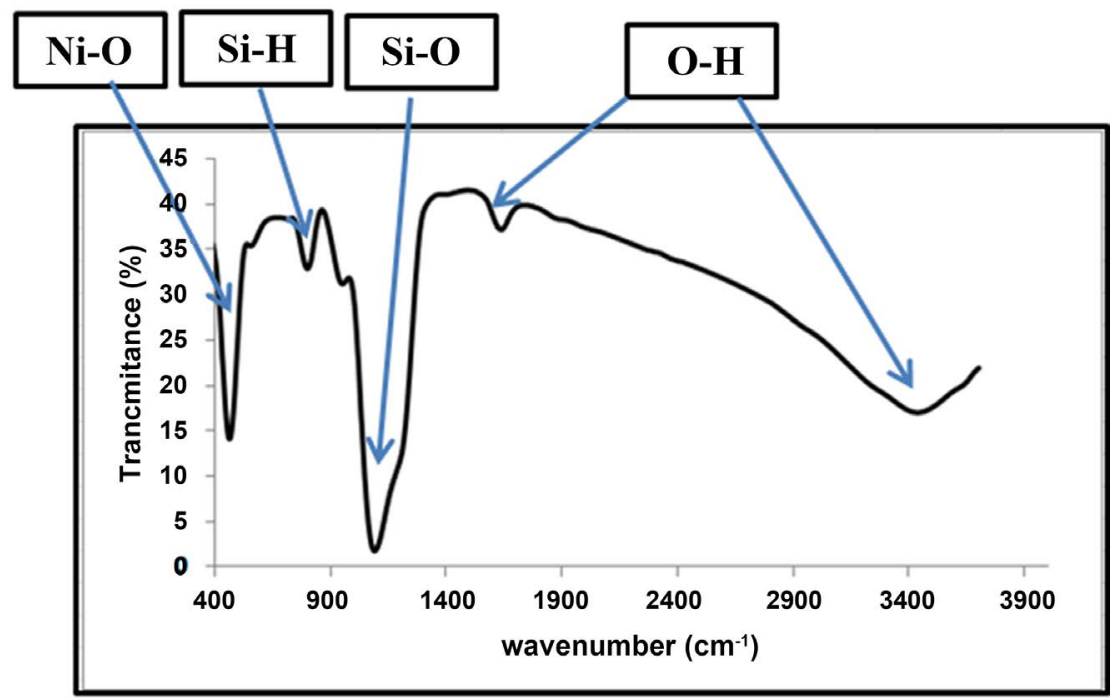

Figure 4. FTIR spectra of (Ni/NPS) nano-composite powder.

Figure 6 shows the Raman-spectra peak at $513 \mathrm{~cm}^{-1}$ for NPS powder which is slightly shifted from 518 to $513 \mathrm{~cm}^{-1}$, that as a result of Ni deposition within the 


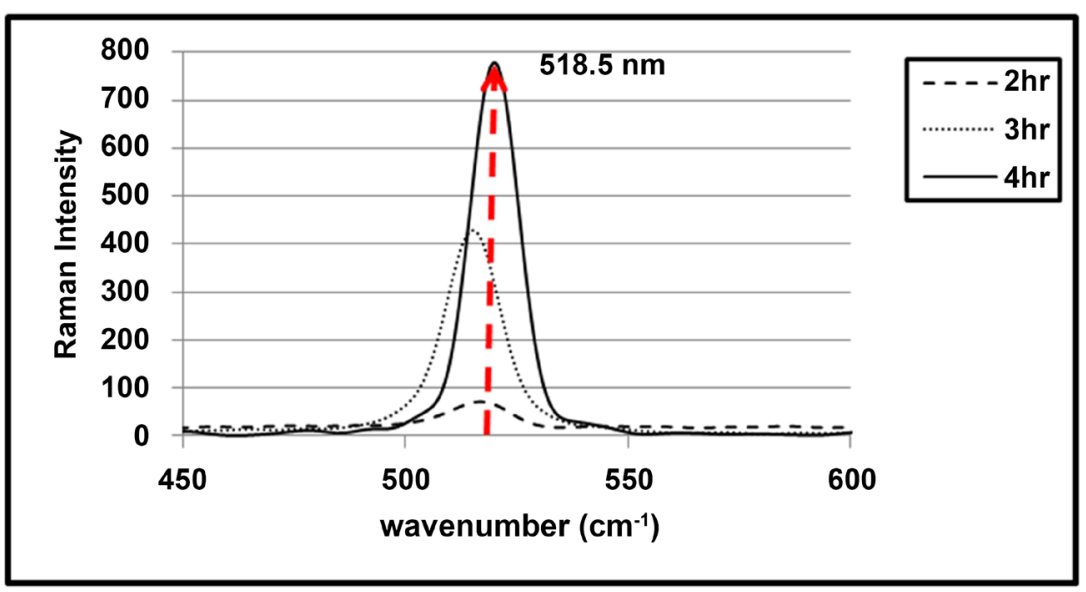

Figure 5. Raman spectra of NPS powder which is prepared at the preparation conditions; 6 weight $\% \mathrm{KOH}$, at different sonication times.

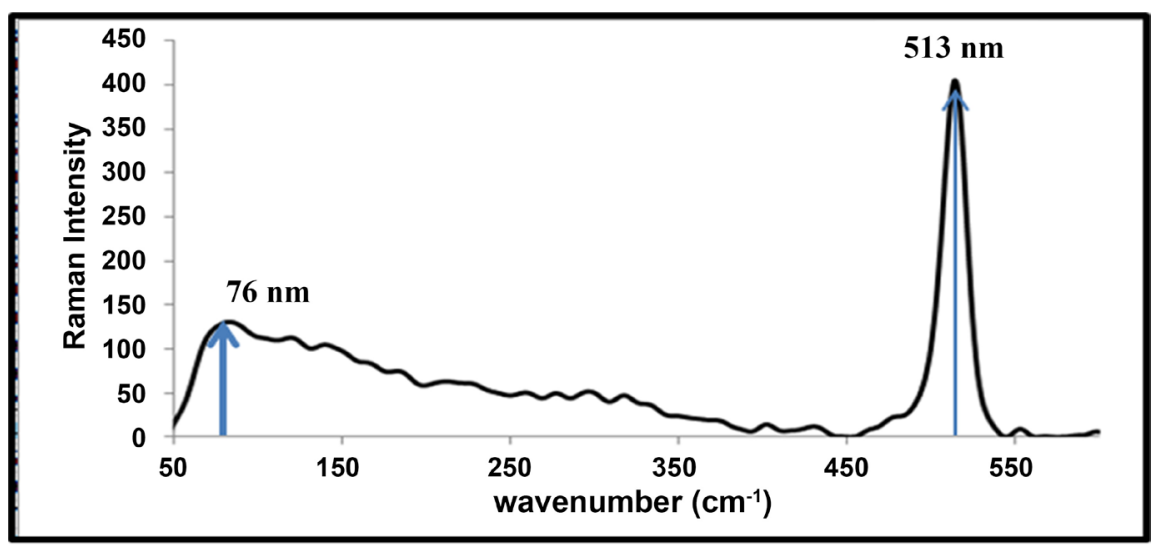

Figure 6. Raman spectra of (Ni/NPS) nano-composite powder.

pores for the nano-composite (Ni/NPS) formation. The broad peak $76 \mathrm{~cm}^{-1}$ is observed, which is corresponding to $\mathrm{NiO}$ formation [16]. $\mathrm{NiO}$ is responsible the shifting process of NPS peak, in addition, it's the base of the (Ni/NPS) composite formation.

\section{- Photoluminescence Analysis}

The photoluminescence properties were obtained using an unfocused argon-ion laser with an excitation wavelength of $340 \mathrm{~nm}$ at room temperature [9]. The PL peak is observed in the visible region. Figure 7 shows the PL spectra present emission peaks centered at two places approximately 663 and $706 \mathrm{~nm}$ [17]. So, there are several optical properties as a result of two emission regions. As shown in Figure 7, the emission zones depend on the preparation conditions. The orange red light emission, which corresponds to $663 \mathrm{~nm}$, is believed to that the quantum confinement effect in the nanostructure of prepared NPS powder. In addition, the dark red light emission corresponds to $706 \mathrm{~nm}$. Figure 7(a) and Figure 7(c) display the higher value of the dark red photoluminescence emission intensity at (6 weight $\% \mathrm{KOH}_{\text {conc }}$ and at sonication time $2 \mathrm{hr}$ ) as a good condition. Noticeable, the same behavior appears as Figure $7(\mathrm{c})$, the sonication time 

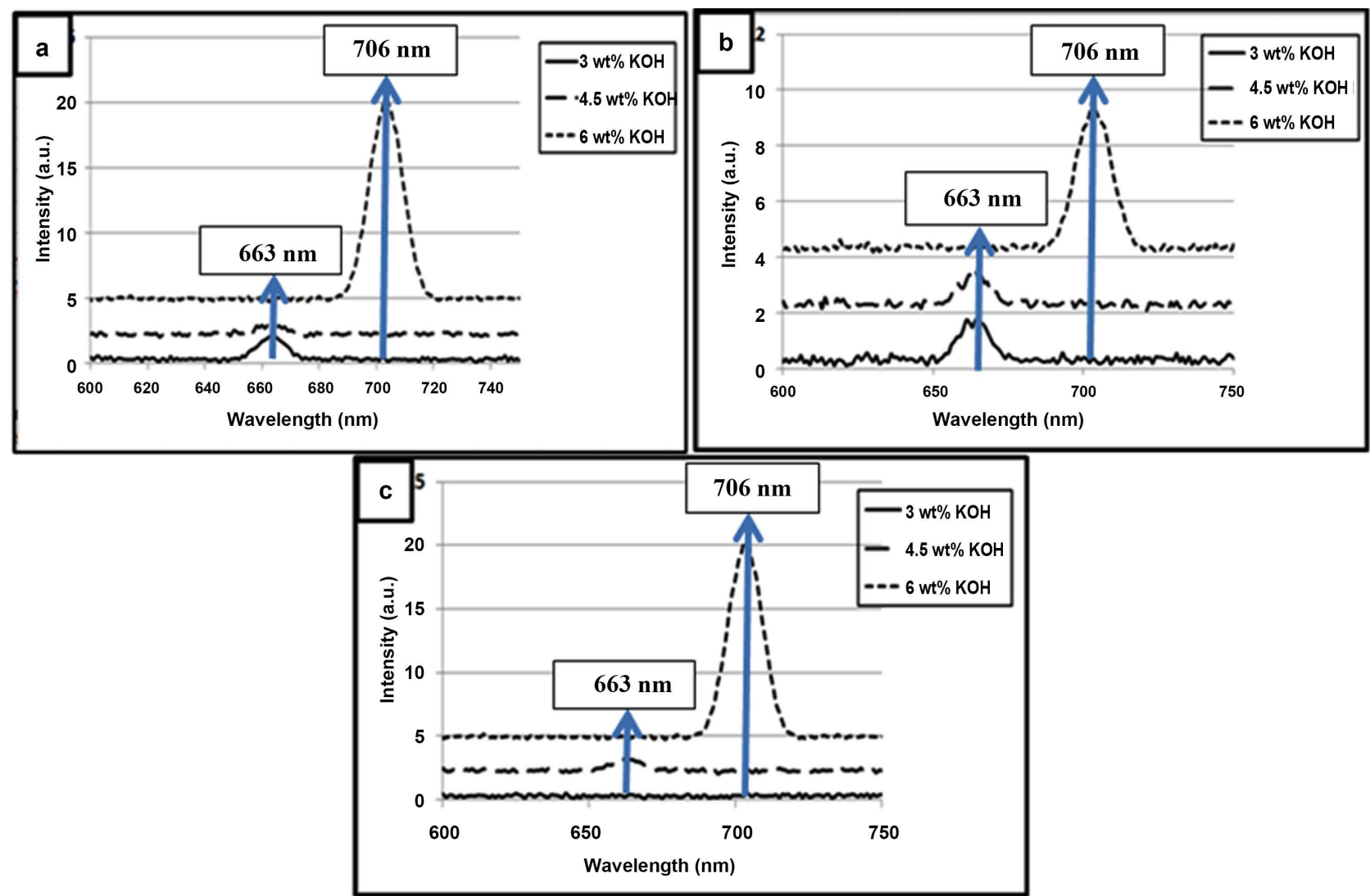

Figure 7. PL spectra of NPS powder at the preparation conditions; 5 gm of commercial silicon powder, 3 weight $\% \mathrm{KOH}$, and at sonication time; (a) $2 \mathrm{hr}$, (b) $3 \mathrm{hr}$, and (c) $4 \mathrm{hr}$.

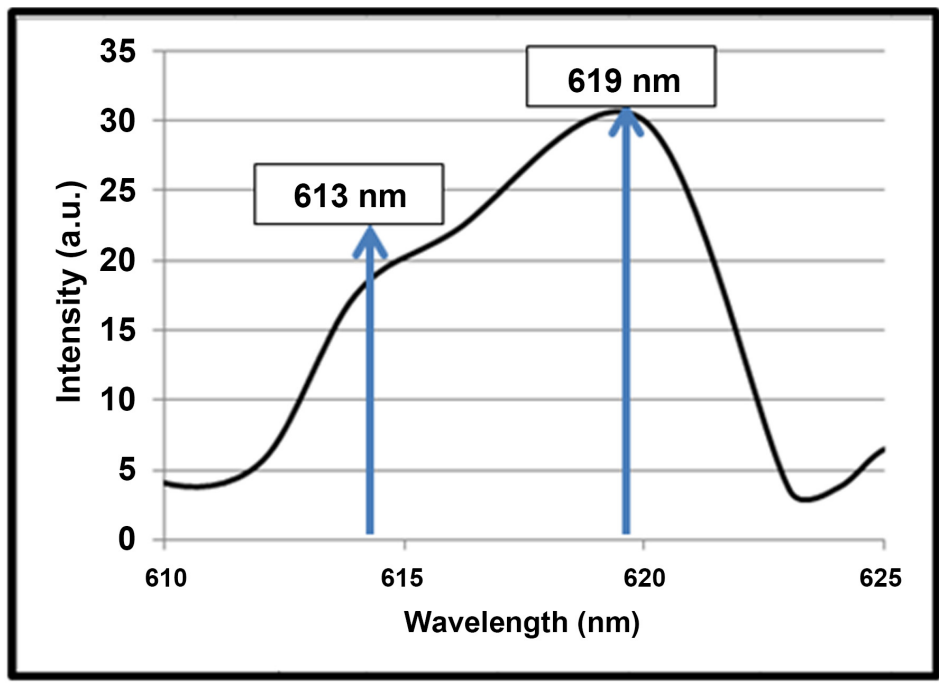

Figure 8. PL spectra of (Ni-NPS) nano-composite powder.

$4 \mathrm{hr}$. On the other hand, Figure 7(b) shows the higher intensity of the orange red photoluminescence emission at both $\mathrm{KOH}_{\text {conc }} 3$ and 4.5 weight\%.

The doping process of the NPS powder with Ni element is the reason of the photoluminescence emission blue shift that corresponds to yellow color. Figure 8 shows the overlapping of two high-intensity photoluminescence emission 
peaks; at 613 and $619 \mathrm{~nm}$. The light emission and its enhancement should be related to the Si-O-bonded nanostructure. The localized state related to $\mathrm{Si}-\mathrm{O}$ bonds and self-trapped excitations in the nanoporous structures is the main origins of the light emission [18] [19]. With the increase of the Ni doping on the NPS surface, it produces Si-O-Ni bonds that agree with the FTIR data. It offers more light-emitting centers and the PL intensity is greatly enhanced. And so we actually managed to get an active material (NPS) for producing several photoluminescence emission ranges, that during the change of the preparation conditions.

\section{Conclusion}

The optical properties of NPS and (Ni/NPS) nanocomposites are investigated in this study. The NPS is prepared using dual reaction (wet alkali chemical etching process and the ultra-sonication technique). In addition, (Ni/NPS) is obtained using the chemical deposition technique. The used techniques are safe, non-expensive, plus it's environmentally friendly. As for the critical factors affecting the preparation process are the $\mathrm{KOH}$ concentration and the sonication time. Also, the presence of a Ni element in the NPS matrix is confirmed by FTIR and Raman spectroscopy. Noticeable, the PL intensity of (Ni/NPS) is about 2 times stronger than that of NPS only. The enhancement of the PL intensity is due to the reaction between Ni ions and NPS surface which contributes to the emission mechanism. So, the NPS material can be used in LED industry, because it has more than one region of PL emission (orange red, dark red and yellow).

\section{Conflicts of Interest}

The author declares no conflicts of interest regarding the publication of this paper.

\section{References}

[1] Jiang, R., Du, X., Sun, W., Han, Z. and Wu, Z. (2015) Enhancement of The Blue Photoluminescence Intensity for The Porous Silicon with $\mathrm{HfO}_{2}$ Filling into Microcavities. Scientific Reports, 5, 15574-15581. https://doi.org/10.1038/srep15574

[2] Kanemitsu, Y., Suzuki, K., Uto, H., Masumoto, Y., Matsumoto, T., Kyushin, S., Higuchi, K. and Matsumoto, H. (1992) Visible Photoluminescence of Silicon Based Nanostructures: Porous Silicon and Small Silicon Based Clusters. Applied Physics Letters, 61, 2446-2448. https://doi.org/10.1063/1.108147

[3] Jern-Ee, D.T., Sheng, C.K. and Isa, M.I.N. (2011) Photoluminescence of Porous Silicon Prepared by Chemical Etching Method. The Malaysian Journal of Analytical Sciences, 2, 227-231.

[4] Amdouni, S., Rahmani, M., Zaïbi, M.A. and Oueslati, M. (2015) Enhancement of Porous Silicon Photoluminescence by Electroless Deposition of Nickel. Journal of Luminescence, 157, 93-97. https://doi.org/10.1016/j.jlumin.2014.08.041

[5] Ayat, M., Belhousse, S., Boarino, L., Gabouze, N., Boukherroub, R. and Kechouane, M. (2014) Formation of Nanostructured Silicon Surfaces by Stain Etching. Nanoscale Research Letters, 9, 482-288. https://doi.org/10.1186/1556-276X-9-482 
[6] Wong, H. (2002) Recent Developments in Silicon Optoelectronic Devices. Microelectronics Reliability, 42, 317-326. https://doi.org/10.1016/S0026-2714(02)00008-2

[7] Russo, L., Colangelo, F., Cioffi, R., Rea, I. and Stefano, L.D. (2011) A Mechanochemical Approach to Porous Silicon Nanoparticles Fabrication. Materials, 4, 1023-1033. https://doi.org/10.3390/ma4061023

[8] Kamath, S.R. and Proctor, A. (1998) Silica Gel from Rice Hull Ash: Preparation and Characterization. Cereal Chemistry Journal, 75, 484-487. https://doi.org/10.1094/CCHEM.1998.75.4.484

[9] Kashyout, A.E.-H. and Nabil, M. (2018) Production of High Throughput Nano-Porous Silicon (NPS) Powder with Different Architectures. Materials Chemistry and Physics, 216, 454-459. https://doi.org/10.1016/j.matchemphys.2018.05.048

[10] Bashiri, H., Karami, M.A. and Nejad, S.M. (2017) Heterojunction Silicon Solar Cells Performance Optimization and Sensitivity Reduction to the Interface Defect States. Materials Research Express, 4, 126308-12619. https://doi.org/10.1088/2053-1591/aa9c91

[11] Jean, T.M.-M. , Delmounm, E.A. and Uintard, P. (1995) Oxide Layer on Silicon Carbide Powder: A FTIR Investigation. Journal of Molecular Structure, 349, 105-108. https://doi.org/10.1016/0022-2860(95)08720-G

[12] Kashyout, A.E.-H., Soliman, H.M.A., Nabil, M. and Bishara, A.A. (2013) Fabrication of Nano-Porous Silicon Using Alkali Etching Process. Materials Letters, 100, 184-187. https://doi.org/10.1016/j.matlet.2012.12.107

[13] Khalifa, M., Atyaoui, M., Hajji, M., Ouertani, R. and Ezzaouia, H. (2013) Purification of Metallurgical-Grade Silicon Powder Via Chemical Attack by Hydrofluoric and Nitric Acids Followed by Thermal Treatment. Materials Science in Semiconductor Processing, 16, 1742-1746. https://doi.org/10.1016/j.mssp.2013.06.006

[14] Rahdar, A., Aliahmad, M. and Azizi, Y. (2015) NiO Nanoparticles: Synthesis and Characterization. Journal of Nanstructures, 5, 145-151.

[15] Nabil, M. and Motaweh, H.A. (2016) Enhanced Thermal Stability of Promising Nano-Porous Silicon Powder. Advances in Nanoparticles, 5, 199-205. https://doi.org/10.4236/anp.2016.54021

[16] Nabil, M., Elnouby, M., Gayeh, N., Sakr, A.H. and Motaweh, H.A. (2017) Enhancement of Porous Silicon Photoluminescence Using (Ni) Treatment. IOP Conference Series: Materials Science and Engineering, 248, 012001-012008. https://doi.org/10.1088/1757-899X/248/1/012001

[17] Cheah, K.W., Chan, T., Lee, W.L., Teng, D., Zheng, W.H. and Wang, Q.M. (1993) Multiple Peak Photoluminescence of Porous Silicon. Applied Physics Letters, 63, 3464-3466. https://doi.org/10.1063/1.110121

[18] Kjeldstad, T., Thøgersen, A., Stange, M., Jensen, I.T., Monakhov, E. and Galeckas, A. (2019) Surface Effects and Optical Properties of Self-Assembled Nanostructured a-Si: Al. Nanomaterials, 9, 1106-1117. https://doi.org/10.3390/nano9081106

[19] Zhang, C., Li, C., Liu, Z., Zheng, J., Xue, C., Zuo, Y., Cheng, B. and Wang, Q. (2013) Enhanced Photoluminescence From Porous Silicon Nanowire Arrays. Nanoscale Research Letters, 8, 277-280. https://doi.org/10.1186/1556-276X-8-277 